\title{
Analysis of color feature extraction techniques for Fish Species Identification
}

\author{
$1^{\text {st }}$ Uéliton de Paula Freitas \\ Faculdade de Computação - FACOM \\ Universidade Federal de Mato Grosso do Sul \\ Campo Grande, Brazil \\ freitas.ueliton@gmail.com
}

\author{
$4^{\text {th }}$ Edson Takashi Matsubara \\ Faculdade de Computação - FACOM \\ Universidade Federal de Mato Grosso do Sul \\ Campo Grande, Brazil \\ edsontm@facom.ufms.br
}

\author{
$2^{\text {nd }}$ Marcio Carneiro Brito Pache \\ Campus Aquidauana \\ Instituto Federal de Mato Grosso do Sul \\ Aquidauana, Brazil \\ marcio.pache@ifms.edu.br \\ $5^{\text {th }}$ José Sabino \\ Universidade Anhaguera - Uniderp \\ Campo Grande, Brazil \\ sabino-jose@uol.com.br
}

\author{
$3^{\text {rd }}$ Wesley Nunes Gonçalves \\ Inovisão \\ Universidade Federal de Mato Grosso do Sul \\ Campo Grande, Brazil \\ wesley.goncalves@ufms.br \\ $6^{\text {th }}$ Diego André Sant'Ana \\ Campus Aquidauana \\ Instituto Federal de Mato Grosso do Sul \\ Aquidauana, Brazil \\ diego.santana@ifms.edu.br
}

\author{
$7^{\text {th }}$ Hemerson Pistori \\ Inovisão \\ Universidade Católica Dom Bosco \\ Campo Grande, Brazil \\ pistori@ucdb.br
}

\begin{abstract}
Color recognition is an important step for computer vision to be able to recognize objects in the most different environmental conditions. Classifying objects by color using computer vision is a good alternative for different color conditions such as the aquarium. In which it is possible to use resources of a smartphone with real-time image classification applications. This paper presents some experimental results regarding the use of five different feature extraction techniques to the problem of fish species identification. The feature extractors tested are the Bag of Visual Words (BoVW), the Bag of Colors (BoC), the Bag of Features and Colors (BoFC), the Bag of Colored Words (BoCW), and the histograms HSV and RGB color spaces. The experiments were performed using a dataset, which is also a contribution of this work, containing 1120 images from fishes of 28 different species. The feature extractors were tested under three different supervised learning setups based on Decision Trees, K-Nearest Neighbors, and Support Vector Machine. From the attribute extraction techniques described, the best performance was BoC using the Support Vector Machines as a classifier with an FMeasure of 0.90 and AUC of 0.983348 with a dictionary size of 2048.
\end{abstract}

Index Terms-Aquarium Dataset, Fish Image Classification, Machine Learning, Point of Interest, Color Descriptor.

\section{INTRODUCTION}

The Brazilian fauna and flora stand out worldwide for their diversity, thus cataloging species of animals and plants is a complicated and arduous task. With such diversity, given a particular animal, even using books and digital databases, it is laborious to identify it. It takes years of study by a professional to specialize in a particular animal. Thus, areas of science, such as artificial intelligence, aim to facilitate these tasks.

Techniques such as the Speeded-up Robust Features (SURF) [1] and Scale-Invariant Feature Transform (SIFT) [2], which describe the points of interest of the images are widely used. This way has been used the Bag Of Visual Words - BoVW [3] in which describing the images based on the regions of interest generated by the SURF. BoVW is a technique based on the Bag of Words, mainly used in the description of texts. As well as techniques that use colors along with other information, the Bag of Features and Colors - BoFC [4] uses BoVWbased concepts by adding color information areas of interest generated by SURF.

Another technique called Bag of Colored Words - BoCW [5], [6] has also been implemented and combines the description of the images supplied by SURF and color information provided by the histogram of color in the HSV space.

Moreover, a technique that uses just color to describe the images, the Wengert's Color Histogram [7] (Bag of Colors - BoC), which when used as a global descriptor uses only color information, creating signatures images and the use of histograms in HSV [8] and RGB [9] colors. Once the image description techniques have been defined, we can learn to classify new images into their respective species.

This paper presents an analysis of computer vision and machine learning techniques to classify fish images through experiments done with attribute extraction techniques in colored images of twenty-eight species of fish on a new dataset totaling 1120 images 1 . The images were obtained employing photographic cameras and smartphone cameras. Initially, there were several fish in the image, but, for the extraction of each species' characteristics, the images have been cropped for just

${ }^{1}$ Available at: https://www.dropbox.com/sh/d8yl5vmuz8ocol2/AADIeJP edVyKIx31_YDrzJIa?dl=0 
one fish per image.

In order to identify the fish species, we evaluate the classifiers based on Decision Trees (C4.5) [10], Support Vector Machine (SVM) [11] and K-Nearest Neighbors (KNN) [12]. The Decision Tree was chosen due to the simplicity of the model used in the classification. The $\mathrm{KNN}$ is widely used to classify images and was used in the performance evaluations of the BoC and BoFC. Now, SVM is also popular in object classification, and an example of their use is found in Rova et al. [13].

Therefore, this paper provides results for the classification of aquarium fish images in which two metrics were used to measure the performance of the classifiers and extractors of attributes. This experiment used the F-Measure (F-Scores) criterion of the best parameters of the classifiers and the Area Under the Curve (AUC) as an auxiliary metric and tiebreaker criterion in the choice of parameters.

\section{RELATED WORK}

Some studies have been found in the literature concerning the classification of fishes. Nery et al. [14] reports that fish classification is not an easy task; according to the same, fish have 47 characteristics that define them, such as color, width, and length. Besides, the images in aquariums are usually obtained with different illuminations making it even more challenging to classify. Using a bayesian classifier and vectors of attributes provided with the mentioned characteristics, the authors presented a classification accuracy higher than $90 \%$ using six species of fish.

Rodrigues [15] used an automatic system based on Principal Component Analysis [16] and the Scale-Invariant Feature Transform (SIFT) for the parameterization of shape, appearance, and movement of species. He used two artificial immunological systems (Artificial Immune Network and Adaptive Radius Immune Algorithm) to group the species' characteristics. It obtained $92 \%$ accuracy using PCA and Adaptive Radius Immune Algorithm with KNN classifier in nine species of fish.

Matai et al. (2010) [17] developed research in order to automate the process of detection and recognition of the Scythe butterflyfish (Prognathodes falcifer) and flag rockfish (Sebastes rubrivinctus). For the detection process, the Viola and Jones-VJ algorithm based in haar-like features was used to make background subtraction reaching $90 \%$ of correct hit ratio for butterflyfish and $49 \%$ to rockfish. Principal Component Analysis-PCA and Scale Invariant Feature Transform-SIFT were used for classification, reaching $100 \%$ of hit ratio, although with just a small part of the dataset.

Researchers in [18] has developed a set-based approach to fish species identification to video captured from uncontrolled underwater environments. The approach consists of the tracking for separation of categories of species during the training and, new images were tracking in the test, and the system got an overall accuracy of $94.6 \%$.

A machine learning approach was developed by Sengar et al. (2017) [19] for the identification of fish quality through
Region of Interest - ROI segmentation of fish eyes and pupil after pesticide exposure. The dataset has 144 images of Indian Rohu (L. rohita) fish in which the proportion of $50 \%$ contains cypermethrin pesticide and $50 \%$ not. From that 144 images, 80 was separated for training consists of 40 samples with pesticide The Random Forest classifier got the best performance of $96.87 \%$ for fish pupil and $93.75 \%$ for the fisheye.

Rathi et al. (2017) [20] got good results using Convolutional Neural Networks-CNN and Image Processing for fish image classification in an underwater environment achieving an accuracy of $96.29 \%$ tested in the Fish4Knowledge dataset containing 27,142 images.

Allken et al. (2018) [21] also applied CNN for the identification of fish specimens but training with synthetic data from Blue Whiting, Atlantic Herring, and Atlantic Mackerel. The automatic system has a classification accuracy of $94 \%$ for synthetic data and 67.2 for real images.

Another CNN-based approach was developed by [22] for fish detection from a computer vision system embedded in an Autonomous Underwater Vehicle (AUV). Since original images were not enough, Data Augmentation was adopted to outperform the training step and algorithm optimization. It was required for time reduction in real-time operation. Thus in the three performed experiments: with Data Augmentation, the better average confidence was 0.65 ; with the Dropout training loss function, they reached 0.28 , and with the refined sum-squared loss function, the prediction reaches a convergent point of 0.27 at 650 iteration time.

Recently, [23] proposed a Deep CNN for automatic fish species identification based on the AlexNet model using four convolutional layers and two fully connected layers. The proposed model was applied for freshwater fish farming images from six species, resulting after data augmentation by zooming, rotation, and flipping in a total of 1334 images. So, they obtained a testing accuracy of $90.48 \%$.

Rauf [24] also take advantage of Deep CNN for automatic identification of fishes species available at the Fish-Pak dataset. It has 941 fish images from six species subdivided into morphological features such as head region, body shape, and fin rays. The best results for a learning rate, 0.001 and momentum 0.9 , were $95.73 \%, 96.02 \%$, and $96.94 \%$ of accuracy, respectively, for the head region, body shape, and fin rays. However, it is essential to mention that the Fish-Pak images were preprocessed, removing the background, and the model uses a small amount of data for deep learning techniques.

Yusup [25] developed a deep learning system in order to identify reefs fishes during real-time operations. The dataset consists of 24 species of reef fishes with a total of 9734 images and the labeling was done with Labeling software. The Yolo has been used for species identification and the best results were reach by Pomacanthus imperator with $90.70 \%$ in the testing accuracy. 


\section{EXPERIMENTS}

For each extractor, were generated dictionaries with different sizes that describe each species of fish. In the case of the HSV and RGB histograms, what was varied was the number of bands in which each color channel was divided. The experiments were done with implementations of the classifiers provided by Scikit-learn.

All the classifiers' parameters were varied to obtain the best results in the classification using cross-validation with ten folds. Therefore, the description of the provided images of each technique was submitted to the classifiers with all parameter variations described below.

\section{A. Variations of Classifier Parameters}

The parameters of the classifiers were varied as follows:

- SVM: linear and RBF kernels were used. According to Chang et al. [26], the linear core performs best for a large set of attributes, fitting into the context of this work. The RBF core can adapt training sets with non-linear attributes that were even inserted into the experiments. The values of $\mathrm{C}$ and $\gamma$ were varied in the space logarithmic correlation corresponding to the values: $\log _{2}^{-5}$ to $\log _{2}^{15}$ for $C$ and $\log _{2}^{-15}$ to $\log _{2}^{3}$ for $\gamma$.

- KNN: the value of $\mathrm{K}$ was varied from 1 to 500 , increasing by one. The metrics used to calculate the distance of the points also varied.

- Decision Tree: as the strategy of dividing each node of the tree, it was utilized the best division or a random one. The division criterion was also varied between entropy and Gini impurity.

All parameter permutations were applied to the dictionary variations of the techniques based on the $\mathrm{BoW}$ and the variations of histogram ranges.

\section{B. Determination of Parameters of Attribute Extractors}

To determine the dictionary's size, that best represents each species' characteristics according to the mentioned extractors, and dictionaries were generated with sizes: 32, 64, 128, 256, 512, 1024, 2048, 4096, and 8192 for BoVW, BoC, BoFC, and $\mathrm{BoCW}$. Because they describe images differently compared to extractors based on BoVW, the number of attributes that describe an image is different in the HSV and RGB histograms.

The variation occurs because the histograms divide each channel from the color space into tracks ranging from 8,16 , 32. The minimum number of tracks has been set in $8(512$ attributes) because for smaller values, the loss of information of color is considerable. The maximum number of tracks is 32 (32,768 attributes) due to hardware limitations found in the computers used in the experiments. The experiments were executed with 10 -fold cross-validation, and ten replicates with all the mentioned parameters in Section A.

\section{Results And Discussion}

The results of the best parameter settings are shown in the following tables.
1) Experiments with BoVW: In Table I it is possible to observe that the dictionary of size 4096 obtained better FMeasure and AUC with the SVM classifier. The SVM parameters to achieve this value were as follows: linear core and C $=24826.608981569752$.

TABLE I

DECISION TREE WITH BoVW.

\begin{tabular}{lll|ll|ll}
\hline Dictionary Sizes & \multicolumn{3}{c}{ SVM } & \multicolumn{2}{c}{ KNN } & \multicolumn{2}{c}{ Decision Tree } \\
& F-Measure & AUC & F-Measure & AUC & F-Measure & AUC \\
\hline 32 & 0.16 & 0.822183 & 0.15 & 0.646302 & 0.18 & 0.571267 \\
64 & 0.17 & 0.735667 & 0.14 & 0.714605 & 0.22 & 0.598646 \\
128 & 0.21 & 0.803140 & 0.16 & 0.705625 & 0.21 & 0.6 \\
256 & 0.31 & 0.833187 & 0.19 & 0.710104 & 0.25 & 0.619818 \\
512 & 0.38 & 0.874362 & 0.20 & 0.750654 & 0.25 & 0.638622 \\
1024 & 0.49 & 0.902782 & $\mathbf{0 . 2 3}$ & $\mathbf{0 . 7 3 9 5 0 6}$ & 0.26 & 0.617659 \\
2048 & 0.57 & 0.927132 & 0.21 & 0.680064 & $\mathbf{0 . 2 6}$ & $\mathbf{0 . 6 3 5 2 4 4}$ \\
4096 & $\mathbf{0 . 5 9}$ & $\mathbf{0 . 9 4 4 1 3 5}$ & 0.20 & 0.650344 & 0.24 & 0.634283 \\
8192 & 0.56 & 0.933591 & 0.14 & 0.560329 & 0.22 & 0.634658 \\
\hline
\end{tabular}

2) Experiments with BoFC: The dictionary size that stood out over the others was the size 2048 with F-Measure equal to 0.8. The result was obtained using the SVM with $\mathrm{C}=$ 8.86516908684, $\gamma=8.08386864682 \mathrm{e}-05$ and RBF kernels. Table [I illustrates the results of F-Measure for variations of dictionary sizes.

TABLE II

COMParison OF BOFC Dictionaries SETS

\begin{tabular}{lll|ll|ll}
\hline Dictionary Size & \multicolumn{3}{c}{ SVM } & \multicolumn{2}{c}{ KNN } & \multicolumn{2}{c}{ Decision Tree } \\
& F-Measure & AUC & F-Measure & AUC & F-Measure & AUC \\
\hline 32 & 0.25 & 0.823231 & 0.24 & 0.799302 & 0.29 & 0.602282 \\
64 & 0.42 & 0.889544 & 0.39 & 0.833239 & 0.40 & 0.661901 \\
128 & 0.56 & 0.929800 & 0.53 & 0.908964 & 0.47 & 0.727013 \\
256 & 0.68 & 0.955215 & 0.58 & 0.860894 & 0.48 & 0.750654 \\
512 & 0.76 & 0.967876 & 0.62 & 0.870247 & 0.53 & 0.751483 \\
1024 & 0.77 & 0.969725 & $\mathbf{0 . 6 3}$ & $\mathbf{0 . 7 8 4 5 3 8}$ & 0.55 & 0.748871 \\
2048 & $\mathbf{0 . 8 0}$ & $\mathbf{0 . 9 7 2 6 0 7}$ & 0.56 & 0.754933 & $\mathbf{0 . 5 6}$ & $\mathbf{0 . 7 4 8 9 9 9}$ \\
4096 & 0.78 & 0.974841 & 0.49 & 0.727618 & 0.53 & 0.758953 \\
8192 & 0.79 & 0.968803 & 0.37 & 0.608176 & 0.49 & 0.733589 \\
\hline
\end{tabular}

3) Experiments with $B o C$ : The dictionary with the best performance with F-Measure were those of sizes 2048 and 8192. Using the AUC's tiebreaking criterion, the dictionary that best describes the species is size 2048. The SVM parameter settings were the following for the best result found: $\mathrm{C}$ $=1635.68097512,=0.0471890060599$, and core RBF. Table [III illustrates the results of F-Measure for size variations of dictionaries.

TABLE III

COMPARISON OF BoC Dictionaries SIZES

\begin{tabular}{lll|ll|ll}
\hline Dictionary Size & \multicolumn{3}{c}{ SVM } & \multicolumn{2}{c}{ KNN } & \multicolumn{2}{c}{ Decision Tree } \\
& F-Measure & AUC & F-Measure & AUC & F-Measure & AUC \\
\hline 32 & 0.77 & 0.966132 & 0.69 & 0.825876 & 0.59 & 0.753064 \\
64 & 0.83 & 0.973133 & 0.75 & 0.857908 & 0.64 & 0.780888 \\
128 & 0.84 & 0.974144 & 0.76 & 0.862319 & 0.62 & 0.800545 \\
256 & 0.85 & 0.977770 & 0.78 & 0.879424 & 0.64 & 0.817035 \\
512 & 0.89 & 0.985639 & 0.80 & 0.880192 & 0.65 & 0.835304 \\
1024 & 0.88 & 0.983281 & 0.82 & 0.889093 & 0.69 & 0.810591 \\
2048 & $\mathbf{0 . 9 0}$ & $\mathbf{0 . 9 8 3 3 4 8}$ & 0.80 & 0.883555 & 0.68 & 0.840596 \\
4096 & 0.88 & 0.979725 & 0.77 & 0.869325 & 0.71 & 0.852780 \\
8192 & $\mathbf{0 . 9 0}$ & $\mathbf{0 . 9 8 1 4 0 4}$ & $\mathbf{0 . 9 0}$ & $\mathbf{0 . 8 6 4 8 1 6}$ & $\mathbf{0 . 7 7}$ & $\mathbf{0 . 8 5 7 7 2 2}$ \\
\hline
\end{tabular}

4) Experiments with BoCW: Because $\mathrm{BoCw}$ is a BoVWderived technique histogram, the number of attributes that each image describes vary in the size of the dictionary used by BoVW and the total bands of the histogram. Thus, for each 
dictionary size, the number of tracks ranges from $2,4,8,16$, and 32. The 512 and 1024 size dictionaries, both with 32 tracks, obtained the same F-Measure score of 0.88 . However, taking the AUC as a tiebreaker, the dictionary of size 1024 was chosen. The best results were obtained by a track range of 32, as shown in Table IV

TABLE IV

COMPARISON OF BOCW DICTIONARY SIZES USING 32 TRACKS.

\begin{tabular}{lll|ll|ll}
\hline Dictionary Size & \multicolumn{2}{c}{ SVM } & \multicolumn{2}{c}{ KNN } & \multicolumn{2}{c}{ Decision Tree } \\
& F-Measure & AUC & F-Measure & AUC & F-Measure & AUC \\
\hline 32 & 0.87 & 0.978157 & 0.66 & 0.772911 & 0.71 & 0.835395 \\
64 & 0.87 & 0.978598 & $\mathbf{0 . 6 8}$ & $\mathbf{0 . 7 6 2 3 1 2}$ & $\mathbf{0 . 7 2}$ & 0.825212 \\
128 & 0.86 & 0.961626 & 0.66 & 0.738020 & $\mathbf{0 . 7 2}$ & $\mathbf{0 . 8 3 8 9 3 7}$ \\
256 & 0.86 & 0.971717 & 0.65 & 0.743238 & 0.71 & 0.822060 \\
512 & $\mathbf{0 . 8 8}$ & $\mathbf{0 . 9 7 2 7 0 9}$ & 0.65 & 0.730703 & 0.72 & 0.840050 \\
1024 & $\mathbf{0 . 8 8}$ & $\mathbf{0 . 9 7 3 6 5 6}$ & 0.63 & 0.697975 & 0.71 & 0.821125 \\
2048 & 0.87 & 0.972574 & 0.59 & 0.650194 & 0.71 & 0.824874 \\
4096 & 0.72 & 0.970887 & 0.27 & 0.669057 & 0.71 & 0.819003 \\
8192 & 0.68 & 0.967433 & 0.19 & 0.590258 & 0.69 & 0.816841 \\
\hline
\end{tabular}

5) Experiments with the HSV Color Histogram: Table V presents the comparison of the results obtained using the color histogram with HSV color space, the best F-Measure obtained was 0.89 along with an AUC equal to 0.981666 using 32 tracks with SVM classifier with RBF core, $C=16833.4006851$ and $\gamma=0.00210510528871$.

TABLE V

COMPARISON OF THE NUMBER OF HSV COLOR HISTOGRAM BANDS.

\begin{tabular}{lll|ll|ll}
\hline Tracks & \multicolumn{2}{c}{ SVM } & \multicolumn{2}{c}{ KNN } & \multicolumn{2}{c}{ Decision Tree } \\
& F-Measure & AUC & F-Measure & AUC & F-Measure & AUC \\
\hline 04 & 0.83 & 0.959969 & 0.80 & 0.898065 & 0.74 & 0.859452 \\
08 & 0.86 & 0.967860 & $\mathbf{0 . 8 6}$ & $\mathbf{0 . 9 1 3 2 1 7}$ & $\mathbf{0 . 8 3}$ & $\mathbf{0 . 8 7 6 2 7 5}$ \\
16 & 0.87 & 0.974043 & 0.77 & 0.883935 & 0.71 & 0.850113 \\
32 & $\mathbf{0 . 8 9}$ & $\mathbf{0 . 9 8 1 6 6 6}$ & 0.70 & 0.851477 & 0.69 & 0.838484 \\
\hline
\end{tabular}

6) Experiments with the RGB Color Histogram: The best F-Measure obtained in the experiments using the RGB color histogram was 0.88 with 16 tracks, as shown in Table VI. The parameters of the SVM classifier were linear core and $C=6.3540113525$.

TABLE VI

COMPARISON OF THE NUMBER OF TRACKS IN THE RGB COLOR HISTOGRAM.

\begin{tabular}{lll|ll|ll}
\hline Tracks & \multicolumn{2}{c}{ SVM } & \multicolumn{2}{c}{ KNN } & \multicolumn{2}{c}{ Decision Tree } \\
& F-Measure & AUC & F-Measure & AUC & F-Measure & AUC \\
\hline 04 & 0.86 & 0.951616 & 0.83 & 0.913880 & 0.73 & 0.876630 \\
08 & 0.87 & 0.963213 & $\mathbf{0 . 8 5}$ & $\mathbf{0 . 9 2 0 6 4 1}$ & $\mathbf{0 . 7 6}$ & $\mathbf{0 . 8 6 3 2 5 1}$ \\
16 & $\mathbf{0 . 8 8}$ & $\mathbf{0 . 9 7 4 3 0 0}$ & 0.81 & 0.908237 & 0.75 & 0.854777 \\
32 & 0.87 & 0.986589 & 0.72 & 0.865965 & 0.70 & 0.844429 \\
\hline
\end{tabular}

The result obtained made use of the dictionaries with higher F-Measure and the optimized classifiers, and the attribute extractors were compared to find out the best classifier. For greater reliability of results, the Friedman hypothesis test [27] was applied to evidence the statistical difference of the results.

Table VII illustrates the performance of the SVM classifier concerning F-Measure and AUC of each attribute extractor, since it was the classifier that obtained the best results. Thus, the statistical tests were performed using the F-Measure of the dictionaries illustrated in Table VII Analyzing the attribute extractors, BoC obtained the best result, followed by RGB and HSV histograms.

TABLE VII

COMPARISON OF THE F-MEASURE METRIC WITH THE USE OF THE SVM CLASSIFIER FOR EACH EXTRACTOR.

\begin{tabular}{llll}
\hline Extractor & Dictionary/Track & F-Measure & AUC \\
\hline BoVW & 4096 & 0.59 & 0.944135 \\
BoC & $\mathbf{2 0 4 8}$ & $\mathbf{0 . 9 0}$ & $\mathbf{0 . 9 8 3 3 4 8}$ \\
BoFC & 2048 & 0.80 & 0.972607 \\
BoCw & $1024 / 16$ tracks & 0.88 & 0.973656 \\
Color Histogram - HSV & 32 tracks & 0.89 & 0.981666 \\
Color Histogram - RGB & 16 tracks & 0.88 & 0.974300 \\
\hline
\end{tabular}

Figure 1 illustrates the box diagram of the F-Measure values for each technique. It is possible to observe that the $\mathrm{BoC}$ attribute extractor and HSV and RGB histograms have FMeasure values around 0.9 .

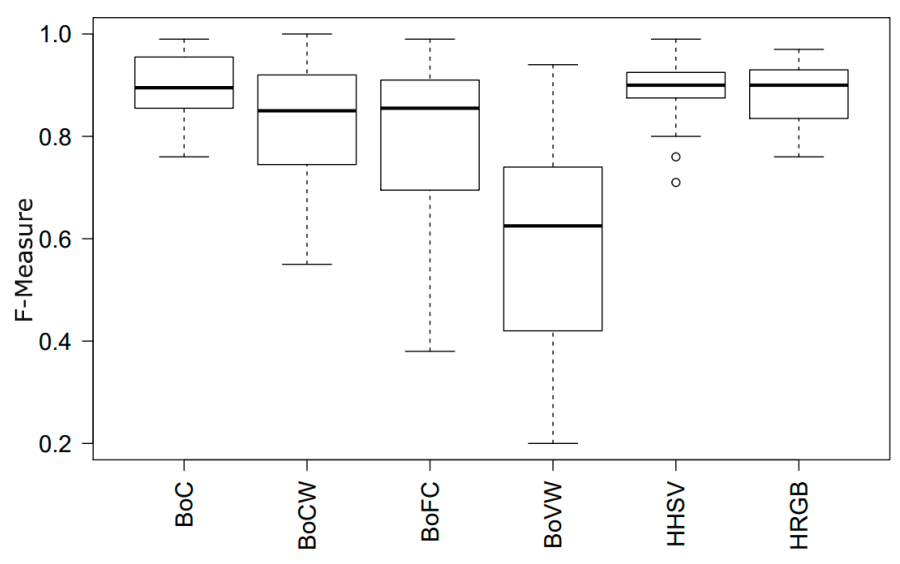

Fig. 1. Box plots of techniques using F-Measure.

The Friedman test obtained a $p$-value of $1.035 \times 10^{-08}$ for F-Measure and $1.275 \times 10^{-13}$ for AUC. Therefore, the posttest [28] was done, and the results are shown in Table VIII] According to the post-test, it is observed that the color-based extractors have a statistical difference with the BoVW, which does not use color to describe the images. As for the colorbased classifiers, according to the Friedman test, they do not present statistical differences between them.

TABLE VIII

Post hoc TEST.

\begin{tabular}{|c|c|c|c|c|c|}
\hline Extractors & $\mathrm{BoFC}$ & $\mathrm{BoC}$ & $\mathrm{BoCW}$ & Histogram HSV & Histogram RGB \\
\hline BoVW & $9.848803 \times 10^{-03}$ & $6.166622 \times 10^{-08}$ & $4.682090 \times 10^{-08}$ & $7.713548 \times 10^{-09}$ & $7.174929 \times 10^{-07}$ \\
\hline $\mathrm{BoFC}$ & - & $1.578712 \times 10^{-01}$ & $1.029825 \times 10^{-01}$ & $7.800627 \times 10^{-02}$ & $3.238798 \times 10^{-01}$ \\
\hline $\mathrm{BoC}$ & - & - & $9.999729 \times 10^{-01}$ & $9.997243 \times 10^{-01}$ & $9.991803 \times 10^{-01}$ \\
\hline $\mathrm{BoCW}$ & - & - & - & $9.999979 \times 10^{-01}$ & $9.943114 \times 10^{-01}$ \\
\hline Histograma HSV & - & - & - & - & $9.868104 \times 10^{-01}$ \\
\hline
\end{tabular}

Figure 2 represents the Friedman post-test box diagram, obtained from the Table VIII As shown, it is possible to observe that the extractors (represented by green boxes) more distant in relation to the $\mathrm{y}=0$ axis are those that present the greatest difference between the interquartile range.

The difference in the performance of BoVW about other techniques is noticeable. This fact is due to the exclusive use 


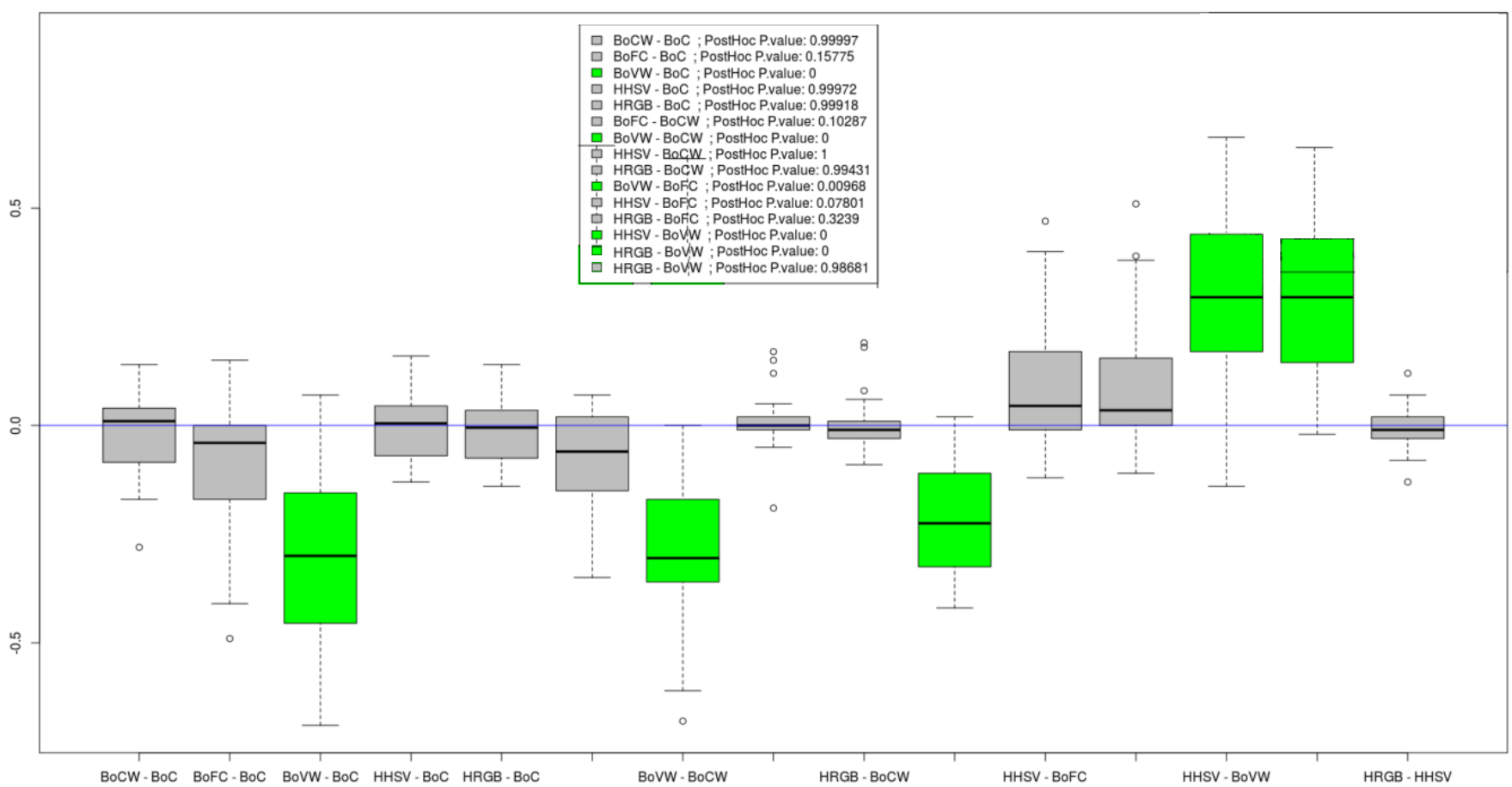

Fig. 2. Friedman post-test box diagram.

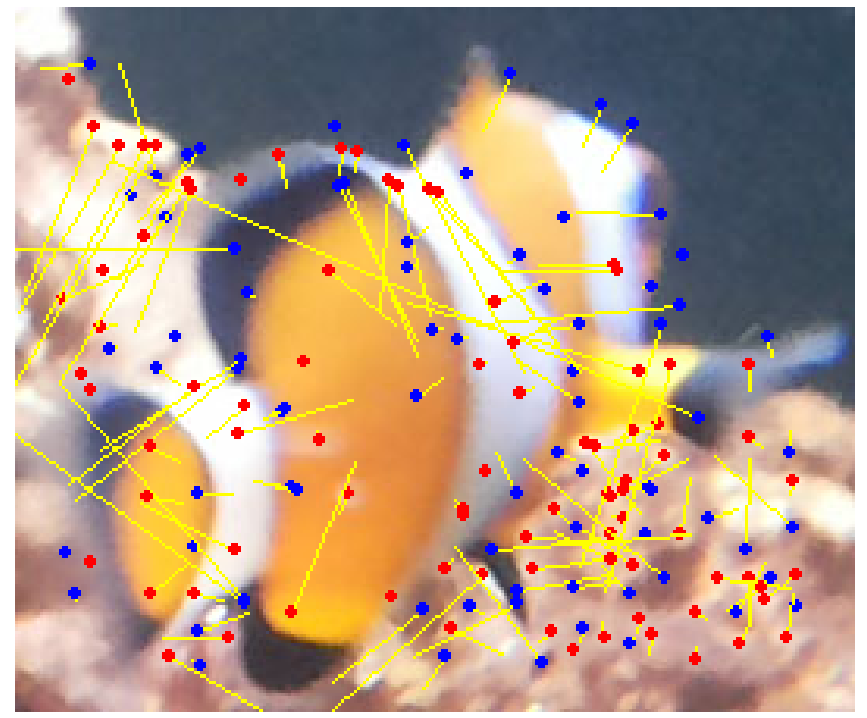

Fig. 3. Example of points of interest found in an image of the Clown fish.

of the cluster of points of interest without taking into account the other characteristics of the fish. The BoVW is based only on points of interest, and they can be found on the fish and/or the background of the image. An example of noise found in one of the images used in the experiments is illustrated in Figure 3 , where it is possible to identify that most of the points of interest were found in the background of the image. Thus, much of the visual words formed are obtained from points that do not belong to the fish, impairing the description.

Nevertheless, the problem of the background of the image has no significant impact on the description of the images when the color is used. This is because, given a set of images relating to a species of fish belonging to a specific aquarium, the background of the images tends to have the same colors since the lighting and the aquarium are the same. Thus, in techniques such as BoC where colors are grouped, it is likely that color is formed corresponding to the average color of the image's background. In the case of $\mathrm{BoC}$, a core representing a color signature corresponding to the background. Moreover, because the training images are from the same aquarium, the average color tends to have a uniform distribution in the description of the images, making it less discriminating in the classification.

The BoFC presented a good result compared to the techniques that use color in the description of the images. Although the same problem was found in the use of points of interest is found in the BoFC, inserting color into the description has attenuated the problem. The fact that the points of interest had color information in their description diminished the impact of the points found in the bottom of the image. For the same reason, cited in the $\mathrm{BoC}$ analysis, and because of this reason, the BoFC obtained better results than the BoVW.

\section{Conclusions}

In this paper, we proposed an aquarium fishes identification method based on point of interest feature extraction, especially color. The fact of grouping the colors of the background of the 
image is evidenced mainly in the $\mathrm{BoCw}$ technique. Observing the Tables I to VI in Section IV, the AUC values from the color information are proportional to the F-Measure values. Initially, for a small number of attributes, the results are close to BoVW due to lower color information compared to the same variety of dictionary size $(32,64, \ldots, 8192)$. However, increasing the amount of bands, the values of F-Measure also increase, evidencing the importance of the color in the description. The color histograms also highlight the importance of color in the classification and the color grouping of the background of the image, since the division of color space into stripes is a way of grouping. For future experiments, we can use other types of attribute extraction techniques and also deep learning.

\section{ACKNOWLEDGMENT}

This work has received financial support from the Universidade Católica Dom Bosco, the Foundation for the Support and Development of Education, Science and Technology from the State of Mato Grosso do Sul - FUNDECT (131/2016) and this study was financed in part by the Coordenação de Aperfeiçoamento de Pessoal de Nível Superior - Brasil (CAPES) - Finance Code 001 and CNPq (National Council for Scientific and Technological Development) through research grants (p. 314902/2018-0). Thanks to Nvidia Corporation for donating the GPU.

\section{REFERENCES}

[1] H. Bay, A. Ess, T. Tuytelaars, and L. Van Gool, "Speededup robust features (surf)," Comput. Vis. Image Underst., vol. 110, no. 3, pp. 346-359, Jun. 2008. [Online]. Available: http: //dx.doi.org/10.1016/j.cviu.2007.09.014

[2] D. G. Lowe, "Distinctive image features from scale-invariant keypoints," Int. J. Comput. Vision, vol. 60, no. 2, pp. 91-110, Nov. 2004. [Online]. Available: http://dx.doi.org/10.1023/B:VISI.0000029664.99615.94

[3] L. F. J. W. G. Csurka, C. Dance and C. Bray, "Visual categorization with bags of keypoints," Workshop on statistical learning in computer vision, vol. 1, pp. 1-22, 2004.

[4] A. Bahri and H. Zouaki, "A surf-color moments for images retrieval based on bag-of-features," in European Journal of Computer Science and Information Technology Vol.1 No.1, pp.11-22, June 2013. Department of Mathematics and computer science, Faculty of Science, El Jadida, Morocco: EAJ, Jun. 2013, pp. 11-22. [Online]. Available: http://www.eajournals.org/journals/european-journal-of-computerscience-and-information-technology-ejcsit/vol-1-issue-1-june-2013/asurf-color-moments-for-images-retrieval-based-on-bag-of-features/

[5] C. S. Joost van de Weijer, "Coloring local feature extraction," European Conference on Computer Vision (ECCV '06), pp. 334-348, 2006.

[6] J. van de Weijer1 and F. S. Khan, "Fusing color and shape for bag-ofwords based object recognition," Computational Color Imaging. CCIW 2013. Lecture Notes in Computer Science, vol. 7786, 2013.

[7] C. Wengert, M. Douze, and H. Jégou, "Bag-of-colors for improved image search," in MM 2011 - 19th ACM International Conference on Multimedia. Scottsdale, United States: ACM, Nov. 2011, pp. 14371440, qUAERO. [Online]. Available: http://hal.inria.fr/inria-00614523

[8] S. Sural, Gang Qian, and S. Pramanik, "Segmentation and histogram generation using the hsv color space for image retrieval," in Proceedings. International Conference on Image Processing, vol. 2, 2002, pp. II-II.

[9] R. Chakravarti and X. Meng, "A study of color histogram based image retrieval," in 2009 Sixth International Conference on Information Technology: New Generations, 2009, pp. 1323-1328.

[10] J. R. Quinlan, C4.5: Programs for Machine Learning. San Mateo, CA: Morgan Kaufmann, 1993.

[11] V. N. Vapnik, "An overview of statistical learning theory," Trans. Neur Netw., vol. 10, no. 5, pp. 988-999, Sep. 1999. [Online]. Available: http://dx.doi.org/10.1109/72.788640
[12] N. Altman, "An introduction to kernel and nearest neighbor nonparametric regression," The American Statistician, vol. 46, pp. 175--185, 1992.

[13] A. Rova, G. Mori, and L. M. Dill, "One fish, two fish, butterfish, trumpeter: Recognizing fish in underwater video," in In IAPR Conference on Machine Vision Applications, 2007.

[14] M. S. Nery, A. M. Machado, M. F. M. Campos, F. L. C. Pádua, R. Carceroni, and J. P. Queiroz-Neto, "Determining the appropriate feature set for fish classification tasks," Graphics, Patterns and Images, SIBGRAPI Conference on, vol. 0, pp. 173-180, 2005.

[15] M. T. A. Rodrigues, "Classifição automática de espécies de peixes baseada em técnicas robustas para extração de caracteristicas e sistemas imunológicos artificiais," Mestrado, Belo Horizonte : Centro Federal de Educacao Tecnologica de Minas Gerais, 2009.

[16] I. Jolliffe, Principal component analysis, ser. Springer series in statistics. Springer-Verlang, 1986. [Online]. Available: http://books. google.com.br/books?id=cN5UAAAAYAAJ

[17] J. Matai, R. Kastner, G. R. Cutter, and D. A. Demer, "Automated techniques for detection and recognition of fishes using computer vision algorithms," 2012.

[18] F. Shafait, A. Mian, M. Shortis, B. Ghanem, P. F. Culverhouse, D. Edgington, D. Cline, M. Ravanbakhsh, J. Seager, and E. S. Harvey, "Fish identification from videos captured in uncontrolled underwater environments," ICES Journal of Marine Science, vol. 73, no. 10, pp. 2737-2746, 07 2016. [Online]. Available: https: //doi.org/10.1093/icesjms/fsw106

[19] N. Sengar, M. K. Dutta, and B. Sarkar, "Computer vision based technique for identification of fish quality after pesticide exposure," International Journal of Food Properties, vol. 20, no. sup2, pp. 21922206, 2017. [Online]. Available: https://doi.org/10.1080/10942912.2017. 1368553

[20] D. Rathi, S. Jain, and S. Indu, "Underwater fish species classification using convolutional neural network and deep learning," 2017 Ninth International Conference on Advances in Pattern Recognition (ICAPR), Dec 2017. [Online]. Available: http://dx.doi.org/10.1109/ICAPR.2017. 8593044

[21] V. Allken, N. O. Handegard, S. Rosen, T. Schreyeck, T. Mahiout, and K. Malde, "Fish species identification using a convolutional neural network trained on synthetic data," ICES Journal of Marine Science, vol. 76, no. 1, pp. 342-349, 10 2018. [Online]. Available: https://doi.org/10.1093/icesjms/fsy147

[22] S. Cui, Y. Zhou, Y. Wang, and L. Zhai, "Fish detection using deep learning," Applied Computational Intelligence and Soft Computing, vol. 2020, p. 3738108, Jan 2020. [Online]. Available: https: //doi.org/10.1155/2020/3738108

[23] M. A. Iqbal, Z. Wang, Z. A. Ali, and S. Riaz, "Automatic fish species classification using deep convolutional neural networks," Wireless Personal Communications, Aug 2019. [Online]. Available: https://doi.org/10.1007/s11277-019-06634-1

[24] S. Z. S. Z. H. S. A. U. R. S. A. C. B. Hafiz Tayyab Raufa, M. Ikram Ullah Lalia, "Visual features based automated identification of fish species using deep convolutional neural networks," Computers and Electronics in Agriculture, vol. 167, no. 105075, 2019.

[25] M. I. I M Yusup and I. Jaya, "Real-time reef fishes identification using deep learning," IOP Conference Series: Earth and Environmental Science, vol. 429, 2019.

[26] C.-J. Chang, Chih-Chung e Lin, LIBSVM: A Library for Support Vector Machines. ACM Transactions on Intelligent Systems and Technology, 2011.

[27] M. Friedman, "A comparison of alternative tests of significance for the problem of m rankings," The Annals of Mathematical Statistics, no. 1, pp. 86-92. [Online]. Available: http://www.jstor.org/stable/2235971

[28] M. Hollander and D. Wolfe, Nonparametric Statistical Methods, ser. A Wiley-Interscience publication. Wiley, 1999. [Online]. Available: http://books.google.com.br/books?id=RJAQAQAAIAAJ 